\title{
A Global Optimization Algorithm for Generalized Quadratic Programming
}

\author{
Hongwei Jiao ${ }^{1,2}$ and Yongqiang Chen ${ }^{3}$ \\ ${ }^{1}$ Department of Mathematics, Henan Institute of Science and Technology, Xinxiang 453003, China \\ ${ }^{2}$ Department of Mathematics, Xidian University, Xi'an 710071, China \\ ${ }^{3}$ Department of Mathematics, Henan Normal University, Xinxiang 453007, China
}

Correspondence should be addressed to Hongwei Jiao; jiaohongwei2013@163.com

Received 13 June 2013; Accepted 13 August 2013

Academic Editor: Md Sazzad Chowdhury

Copyright (C) 2013 H. Jiao and Y. Chen. This is an open access article distributed under the Creative Commons Attribution License, which permits unrestricted use, distribution, and reproduction in any medium, provided the original work is properly cited.

\begin{abstract}
We present a global optimization algorithm for solving generalized quadratic programming (GQP), that is, nonconvex quadratic programming with nonconvex quadratic constraints. By utilizing a new linearizing technique, the initial nonconvex programming problem (GQP) is reduced to a sequence of relaxation linear programming problems. To improve the computational efficiency of the algorithm, a range reduction technique is employed in the branch and bound procedure. The proposed algorithm is convergent to the global minimum of the (GQP) by means of the subsequent solutions of a series of relaxation linear programming problems. Finally, numerical results show the robustness and effectiveness of the proposed algorithm.
\end{abstract}

\section{Introduction}

In this paper, we consider the following generalized quadratic programming problem:

$$
\begin{array}{ll}
(\mathrm{GQP}): \min & g_{0}(x)=x^{T} Q_{0} x+d_{0}^{T} x \\
\text { s.t. } & g_{i}(x)=x^{T} Q_{i} x+d_{i}^{T} x \leq b_{i}, \quad i=1, \ldots, m, \\
& x \in X^{0}=\left\{x \in R^{n}: l^{0} \leq x \leq u^{0}\right\},
\end{array}
$$

where $Q_{i} \in R^{n \times n}(i=0,1, \ldots, m)$ are all symmetric matrices, $d_{0}, d_{i} \in R^{n}, b_{i} \in R, i=1, \ldots, m ; l^{0}=\left(l_{1}^{0}, \ldots, l_{n}^{0}\right)^{T}, u^{0}=$ $\left(u_{1}^{0}, \ldots, u_{n}^{0}\right)^{T}$.

Generalized quadratic programming problems are worthy of study, and it is not only because they have broad applications in heat exchanger network design engineering, financial management, statistics, optimal controllers, and so on $[1,2]$, but also because many other nonlinear problems can be transformed into this form [3,4], and we often approximate nonlinear programming via solution of a series of quadratic programming problems. In addition, from a research point of view, these problems exist as significant theoretical and computational challenges. This is mainly because these problems are global optimization problem; that is, they are known to generally possess multiple local optimal solutions that are not globally optimal. So it is necessary to establish good solution algorithm for generalized quadratic programming problem (GQP).

In the last decades, many solution methods have been developed for globally solving special form and general form of the (GQP). For examples, when constraint condition is a box constraint, a branch-and-cut algorithm [5] has been proposed by Vandenbussche and Nemhauser; when each function $g_{i}(x)(i=1, \ldots, m)$ is a linear function, that is, $\mathrm{GQP}$ is an indefinite quadratic programming with linear constraints, then two efficient algorithms $[6,7]$ have been available; when each function $g_{i}(x)(i=1, \ldots, m)$ is a convex function, that is, GQP is one nonconvex quadratic programming with convex constraints, then two feasible algorithms $[8,9]$ have been presented for globally solving the GQP; by utilizing lagrangian underestimates and the interval newton method, Voorhis [10] has proposed a global optimization algorithm for generalized quadratic programming; by applying a reformulation linearization technique, Sherali and Tuncbilek [11] have presented a feasible method 
for polynomial programming problems. In addition, Qu et al. [12] Shen and Liu [13] have proposed two different branch and bound algorithms for generalized quadratic programming problem using linearizing method, respectively.

In this paper, we will present a global optimization algorithm for solving the (GQP) by combining branch and bound operation with a range reduction technique. The main features of our algorithm are as follows. (1) A new linearizing technique is presented; utilize the technique to systematically convert the GQP problem into a series of relaxation linear programming problems, and the solutions of these converted problems can infinitely approximate the global optimum of the original GQP problem by a successive refinement process. (2) The proposed linearization techniques for generating relaxation linear programming problems are embedded within a branch and bound scheme without increasing new variables and constraints. (3) The proposed relaxation linear programming for the GQP can be easily solved, which is more convenient in computation than the relaxation convex programming; thus, any effective linear programming algorithm can be used to solve this problem. (4) To accelerate the convergent speed of the proposed algorithm, a range reduction technique is applied in the proposed branch and bound procedure, which provides a theoretical possibility to delete a large part of the currently investigated region where there exists no global optimal solution of the (GQP). (5) Combing the new linearizing technique, branch and bound scheme, and range reduction technique, a branch-reductionbound algorithm is presented. Finally, numerical results show that the proposed method can globally solve all test problems in obtaining global optimal solutions within a given tolerance error.

The remainder of this paper is organized as follows. The next section describes the linearizing method and the relaxation linear programming of the original problem is established. Section 3 states the branch-reductionbound algorithm and its convergence. In Section 4 numerical experimental results are given. Finally, the conclusions are presented.

\section{Relaxation Linear Programming}

The principal structure in the development of a solution procedure for solving the (GQP) is the construction of lower bounds for this problem, as well as for its partitioned subproblems. A lower bound on the solution of the (GQP) and its partitioned subproblems can be obtained by solving a relaxation linear programming of the (GQP). In order to generate the relaxation linear programming, the proposed strategy is that underestimates each quadratic function $g_{i}(x)$ $(i=0,1, \ldots, m)$ with a linear function.

In the following, we assume that $X^{k}=\left\{x \in R^{n} \mid l^{k} \leq x \leq\right.$ $\left.u^{k}\right\} \subseteq X^{0}$ with $l^{k}=\left(l_{1}^{k}, \ldots, l_{n}^{k}\right)^{T}, u^{k}=\left(u_{1}^{k}, \ldots, u_{n}^{k}\right)^{T}$. Let $\lambda_{i}$ be the minimum eigenvalues of the matrix $Q_{i}$. Select $\theta_{i}$ such that

$$
\theta_{i}= \begin{cases}0, & \text { if } \lambda_{i} \geq 0 \\ \left|\lambda_{i}\right|+\rho, & \text { if } \lambda_{i}<0\end{cases}
$$

where $\rho$ is a small positive number.
For convenience, for any $x \in X^{k}$, for each $i=0,1, \ldots, m$, define the function

$$
\begin{aligned}
g_{i}^{L}(x)= & {\left[d_{i}+2\left(Q_{i}+\theta_{i} I\right)^{T} u^{k}-2 \theta_{i} u^{k}\right]^{T} x } \\
& +2 \theta_{i}\left(u^{k}\right)^{T} l^{k}-\theta_{i}\left(l^{k}\right)^{T}\left(l^{k}\right) \\
& -\left(u^{k}\right)^{T}\left(Q_{i}+\theta_{i} I\right) u^{k} .
\end{aligned}
$$

Theorem 1. For each $i=0,1, \ldots, m$, for all $x \in X^{k}=\{x \in$ $\left.R^{n} \mid l^{k} \leq x \leq u^{k}\right\} \subseteq X^{0}$, by the definition of $g_{i}(x)$ and $g_{i}^{L}(x)$, we have the following conclusions:

(i) $g_{i}^{L}(x) \leq g_{i}(x)$;

(ii) $\left\|g_{i}(x)-g_{i}^{L}(x)\right\| \rightarrow 0$ as $\left\|u^{k}-l^{k}\right\| \rightarrow 0$.

Proof. (1) For each $j=1, \ldots, n$, by the mean value theorem, for the function $x_{j}^{2}$ over the interval $\left[l_{j}^{k}, u_{j}^{k}\right]$, we have

$$
\begin{aligned}
x_{j}^{2} & =2 \xi_{j}^{k}\left(x_{j}-l_{j}^{k}\right)+\left(l_{j}^{k}\right)^{2} \\
& \leq 2 u_{j}^{k}\left(x_{j}-l_{j}^{k}\right)+\left(l_{j}^{k}\right)^{2} \\
& =2 u_{j}^{k} x_{j}-2 u_{j}^{k} l_{j}^{k}+\left(l_{j}^{k}\right)^{2},
\end{aligned}
$$

where $\xi_{j}^{k}=\alpha l_{j}^{k}+(1-\alpha) u_{j}^{k}, \alpha \in[0,1]$.

From the definition of the function $g_{i}^{L}(x)$ and the equivalent deformation of the function $g_{i}(x)$, we can get that

$$
\begin{aligned}
g_{i}(x)= & x^{T} Q_{i} x+d_{i}^{T} x \\
= & \left(u^{k}-x\right)^{T}\left(Q_{i}+\theta_{i} I\right)\left(u^{k}-x\right) \\
& +d_{i}^{T} x-\theta_{i} \sum_{j=1}^{n} x_{j}^{2}+2\left(u^{k}\right)^{T} \\
& \times\left(Q_{i}+\theta_{i} I\right) x-\left(u^{k}\right)^{T}\left(Q_{i}+\theta_{i} I\right) u^{k} \\
\geq & d_{i}^{T} x-\theta_{i} \sum_{j=1}^{n} x_{j}^{2}+2\left(u^{k}\right)^{T} \\
& \times\left(Q_{i}+\theta_{i} I\right) x-\left(u^{k}\right)^{T}\left(Q_{i}+\theta_{i} I\right) u^{k} \\
\geq & d_{i}^{T} x+\theta_{i} \sum_{j=1}^{n}\left[-2 u_{j}^{k} x_{j}+2 u_{j}^{k} l_{j}^{k}-\left(l_{j}^{k}\right)^{2}\right] \\
& +2\left(u^{k}\right)^{T}\left(Q_{i}+\theta_{i} I\right) x-\left(u^{k}\right)^{T}\left(Q_{i}+\theta_{i} I\right) u^{k} \\
= & {\left[d_{i}+2\left(Q_{i}+\theta_{i} I\right)^{T} u^{k}-2 \theta_{i} u^{k}\right]^{T} x } \\
& +2 \theta_{i}\left(u^{k}\right)^{T} l^{k}-\theta_{i}\left(l^{k}\right)^{T}\left(l^{k}\right) \\
& -\left(u^{k}\right)^{T}\left(Q_{i}+\theta_{i} I\right) u^{k} \\
= & g_{i}^{L}(x) .
\end{aligned}
$$


That is,

$$
g_{i}^{L}(x) \leq g_{i}(x), \quad i=0,1, \ldots, m
$$

(2) By the definition of the function $g_{i}^{L}(x)$ and the proof of conclusion (i), we have

$$
\begin{aligned}
& \left\|g_{i}(x)-g_{i}^{L}(x)\right\| \\
& =\| x^{T} Q_{i} x+d_{i}^{T} x \\
& -\left\{\left[d_{i}+2\left(Q_{i}+\theta_{i} I\right)^{T} u^{k}-2 \theta_{i} u^{k}\right]^{T} x\right. \\
& +2 \theta_{i}\left(u^{k}\right)^{T} l^{k}-\theta_{i}\left(l^{k}\right)^{T}\left(l^{k}\right) \\
& \left.-\left(u^{k}\right)^{T}\left(Q_{i}+\theta_{i} I\right) u^{k}\right\} \| \\
& =\|\left[\left(u^{k}-x\right)^{T}\left(Q_{i}+\theta_{i} I\right)\left(u^{k}-x\right)\right. \\
& +d_{i}^{T} x-\theta_{i} \sum_{j=1}^{n} x_{j}^{2}+2\left(u^{k}\right)^{T} \\
& \left.\times\left(Q_{i}+\theta_{i} I\right) x-\left(u^{k}\right)^{T}\left(Q_{i}+\theta_{i} I\right) u^{k}\right] \\
& -\left\{\left[d_{i}+2\left(Q_{i}+\theta_{i} I\right)^{T} u^{k}-2 \theta_{i} u^{k}\right]^{T} x\right. \\
& +2 \theta_{i}\left(u^{k}\right)^{T} l^{k}-\theta_{i}\left(l^{k}\right)^{T}\left(l^{k}\right) \\
& \left.-\left(u^{k}\right)^{T}\left(Q_{i}+\theta_{i} I\right) u^{k}\right\} \| \\
& =\|\left(u^{k}-x\right)^{T}\left(Q_{i}+\theta_{i} I\right)\left(u^{k}-x\right) \\
& +\theta_{i} \sum_{j=1}^{n}\left[2 u_{j}^{k} x_{j}-2 u_{j}^{k} l_{j}^{k}+\left(l_{j}^{k}\right)^{2}-x_{j}^{2}\right] \| \\
& =\|\left(u^{k}-x\right)^{T}\left(Q_{i}+\theta_{i} I\right)\left(u^{k}-x\right) \\
& +\theta_{i} \sum_{j=1}^{n}\left(x_{j}-l_{j}^{k}\right)\left(u_{j}^{k}-x_{j}+u_{j}^{k}-l_{j}^{k}\right) \| \\
& \leq\left\|\left(u^{k}-x\right)^{T}\left(Q_{i}+\theta_{i} I\right)\left(u^{k}-x\right)\right\| \\
& +\theta_{i}\left\|\sum_{j=1}^{n}\left(x_{j}-l_{j}^{k}\right)\left(u_{j}^{k}-x_{j}+u_{j}^{k}-l_{j}^{k}\right)\right\|
\end{aligned}
$$

$$
\begin{aligned}
\leq & \left\|\left(u^{k}-x\right)^{T}\left(Q_{i}+\theta_{i} I\right)\left(u^{k}-x\right)\right\| \\
& +\theta_{i}\left\|\left(x-l^{k}\right)^{T}\left(u^{k}-x\right)\right\| \\
& +\theta_{i}\left\|\left(x-l^{k}\right)^{T}\left(u^{k}-l^{k}\right)\right\| \\
\leq & \left\|\left(u^{k}-l^{k}\right)^{T}\left(Q_{i}+\theta_{i} I\right)\left(u^{k}-l^{k}\right)\right\| \\
& +2 \theta_{i}\left\|\left(u^{k}-l^{k}\right)^{T}\left(u^{k}-l^{k}\right)\right\| \\
= & \left\|Q_{i}+\theta_{i} I\right\|\left\|u^{k}-l^{k}\right\|^{2}+2 \theta_{i}\left\|u^{k}-l^{k}\right\|^{2} .
\end{aligned}
$$

Since

$$
\begin{array}{r}
\left\|Q_{i}+\theta_{i} I\right\|\left\|u^{k}-l^{k}\right\|^{2}+2 \theta_{i}\left\|u^{k}-l^{k}\right\|^{2} \longrightarrow 0 \\
\text { as }\left\|u^{k}-l^{k}\right\| \longrightarrow 0,
\end{array}
$$

we have

$$
\left\|g_{i}(x)-g_{i}^{L}(x)\right\| \longrightarrow 0 \text { as }\left\|u^{k}-l^{k}\right\| \longrightarrow 0 .
$$

The proof is complete.

By Theorem 1, we can construct the corresponding approximation relaxation linear programming (RLP) of the (GQP) in $X^{k}$ as follows:

$$
\begin{aligned}
\operatorname{RLP}\left(X^{k}\right): \min & g_{0}^{L}(x) \\
\text { s.t. } & g_{i}^{L}(x) \leq b_{i}, \quad i=1, \ldots, m, \\
& x \in X^{k}=\left\{x: l^{k} \leq x \leq u^{k}\right\},
\end{aligned}
$$

where

$$
\begin{aligned}
g_{i}^{L}(x)= & {\left[d_{i}+2\left(Q_{i}+\theta_{i} I\right)^{T} u^{k}-2 \theta_{i} u^{k}\right]^{T} x } \\
& +2 \theta_{i}\left(u^{k}\right)^{T} l^{k}-\theta_{i}\left(l^{k}\right)^{T}\left(l^{k}\right) \\
& -\left(u^{k}\right)^{T}\left(Q_{i}+\theta_{i} I\right) u^{k} .
\end{aligned}
$$

Based on the relaxation linear programming, every feasible point of the (GQP) in subdomain $X^{k}$ is feasible to the (RLP); and the objective function value of the (RLP) is less than or equal to that of (GQP) over the partitioned set $X^{k}$. Thus, the $\operatorname{RLP}\left(X^{k}\right)$ provides a valid lower bound for the solution of the GQP $\left(X^{k}\right)$.

\section{Algorithm and Its Convergence}

In this section, a branch-reduction-bound algorithm is proposed for globally solving the (GQP). To find a global optimal solution, the main computation is to solve a sequence of relaxation linear programming problems over partitioned 
subsets of $X^{0}$. Furthermore, in order to improve the computational efficiency of the proposed algorithm, a range reduction technique is employed to enhance the solution procedure.

The proposed algorithm is based on partitioning the set $X^{0}$ into subhyperrectangles, and each subhyperrectangle is associated with a node of the branch and bound tree, and each node is associated with a relaxation linear programming problem in corresponding subhyperrectangle. Therefore, at any stage $k$ of the algorithm, assume that we obtain a collection of active nodes denoted by $\Delta_{k}$, say, each is associated with a hyperrectangle $X \subseteq X^{0}$, for all $X \in \Delta_{k}$. For each such node $X$, compute a corresponding lower bound $\mathrm{LB}(X)$ of the GQP by solving the RLP, so that the lower bound of optimal value for the (GQP) over the $X^{0}$ at iteration $k$ is updated by $\mathrm{LB}_{k}=$ $\min \left\{\operatorname{LB}(X), \forall X \in \Delta_{k}\right\}$. Until the solution of the (RLP) turns out to be feasible to the (GQP), we renew the upper bound $\bar{g}$ if necessary. Then, the set of the active nodes $\Delta_{k}$ will satisfy $\operatorname{LB}(X)<\bar{g}$, for all $X \in \Delta_{k}$, for each iteration $k$. We now select an active node to partition its associated hyperrectangle into two subhyperrectangles according to the following branching rule, and calculate the lower bounds for each new node as before. Upon fathoming any nonimproving nodes, we get a set of active nodes for the next iteration, and this process is repeated until termination condition is satisfied.

The critical factor in guaranteeing convergence to a global optimum is the choice of a suitable branching rule. In this paper, we will choose a simple and standard bisection rule. This rule is sufficient to ensure convergence since it drives all the intervals to zero for all variables. This branching rule is stated as follows. Consider any node subproblem identified by the rectangle $X^{\prime}=\left[\underline{x}^{\prime}, \bar{x}^{\prime}\right] \subseteq X^{0}$. Let $q=\operatorname{argmax}\left\{\bar{x}_{i}^{\prime}-\underline{x}_{i}^{\prime}\right.$ : $i=1,2, \ldots, n\}$, and partition $X^{\prime}$ into two subrectangles $X_{1}^{\prime}$ and $X_{2}^{\prime}$ by bisection the interval $\left[\underline{x}_{q}^{\prime}, \bar{x}_{q}^{\prime}\right]$ into the subintervals $\left[\underline{x}_{q}^{\prime},\left(\underline{x}_{q}^{\prime}+\bar{x}_{q}^{\prime}\right) / 2\right]$ and $\left[\left(\underline{x}_{q}^{\prime}+\bar{x}_{q}^{\prime}\right) / 2, \bar{x}_{q}^{\prime}\right]$.

3.1. Range Reduction Technique. To improve computational efficiency of the proposed algorithm, a range reduction technique proposed in $[13,14]$ is applied in the algorithm, which is reformulated as the following Theorems 2 and 3. In iteration $k$, we will check whether or not there exists global optimal solution of the $\mathrm{GQP}\left(X^{0}\right)$ over $X$. The proposed technique aims at reducing the rectangle $X$ without deleting any global optimal solution of the $\mathrm{GQP}\left(X^{0}\right)$ over $X$. For convenience, for all $x \in X=\left\{x \in R^{n} \mid l \leq x \leq u\right\} \subseteq X^{0}$ with $l=\left(l_{1}, l_{2}, \ldots, l_{n}\right)^{T}$ and $u=\left(u_{1}, u_{2}, \ldots, u_{n}\right)^{T}$. Without loss of generality, we rewrite the $\operatorname{RLP}(X)$ as the following form:

$$
\begin{aligned}
\operatorname{RLP}(X): \min & g_{0}^{L}(x), \\
\text { s.t. } & g_{i}^{L}(x) \leq b_{i}, \quad i=1, \ldots, m, \\
& x \in X=\{x: l \leq x \leq u\},
\end{aligned}
$$

where

$$
g_{i}^{L}(x)=\sum_{j=1}^{n} c_{i j} x_{j}+\delta_{i}, \quad i=0,1, \ldots, m .
$$

Let $\bar{g}$ be a known upper bound of the optimal value of the $\operatorname{GQP}\left(X^{0}\right)$, and set

$$
\begin{gathered}
\mathrm{RLB}_{i}=\sum_{j=1, c_{i j}>0}^{n} c_{i j} l_{j}+\sum_{j=1, c_{i j}<0}^{n} c_{i j} u_{j}+\delta_{i}, \\
i=0,1, \ldots, m, \\
R g_{i p}^{L}=\sum_{j=1, j \neq p, c_{i j}>0}^{n} c_{i j} l_{j}+\sum_{j=1, j \neq p, c_{i j}<0}^{n} c_{i j} u_{j}+\delta_{i}, \\
i=0,1, \ldots, m, p=1, \ldots, n, \\
\gamma_{p}=\frac{\left(\bar{g}-R g_{0 p}^{L}\right)}{c_{0 p}}, \quad p=1, \ldots, n, \\
\mu_{i p}=\frac{\left(b_{i}-R g_{i p}^{L}\right)}{c_{i p}}, \quad i=1, \ldots, m, p=1, \ldots, n .
\end{gathered}
$$

Theorem 2. For any subrectangle $X \subseteq X^{0}$, the following conclusions hold.

(i) If $R L B_{0}>\bar{g}$, then there exists no global optimal solution of the $G Q P\left(X^{0}\right)$ over $X$.

(ii) If $R L B_{0} \leq \bar{g}$, then, for each $p \in\{1,2, \ldots, n\}$, if $c_{0 p}>$ 0 , then there does not exist global optimal solution of the $G Q P\left(X^{0}\right)$ over $\overline{\bar{X}}=\left(\overline{\bar{X}}_{j}\right)_{n \times 1}$; if $c_{0 p}<0$, there is no global optimal solution of the $G Q P\left(X^{0}\right)$ over $\underline{X}=$ $\left(\underline{X}_{j}\right)_{n \times 1}$, where

$$
\begin{aligned}
& \overline{\bar{X}}_{j}= \begin{cases}X_{j}, & j \neq p, j=1, \ldots, n, \\
\left(\gamma_{p}, u_{p}\right] \bigcap X_{p}, & j=p ;\end{cases} \\
& \underline{X}_{j}= \begin{cases}X_{j}, & j \neq p, j=1, \ldots, n, \\
{\left[l_{p}, \gamma_{p}\right) \bigcap X_{p},} & j=p .\end{cases}
\end{aligned}
$$

Proof. (i) If $\mathrm{RLB}_{0}>\bar{g}$, then for all $x \in X, g_{0}^{L}(x) \geq \mathrm{RLB}_{0}>\bar{g}$; that is,

$$
g_{0}(x) \geq g_{0}^{L}(x) \geq \mathrm{RLB}_{0}>\bar{g}
$$

Therefore, there exists no global optimal solution of the $\mathrm{GQP}\left(X^{0}\right)$ over $X$.

(ii) If $\mathrm{RLB}_{0} \leq \bar{g}$, then, for each $p \in\{1, \ldots, n\}$, if $c_{0 p}>0$, for all $x \in \overline{\bar{X}}$, we have $x_{p}>\gamma_{p}$; that is,

$$
x_{p}>\frac{\bar{g}-R g_{0 p}^{L}}{c_{0 p}} \text { or } \quad c_{0 p} x_{p}>\bar{g}-R g_{0 p}^{L} \text {. }
$$


Therefore, we get that

$$
\begin{aligned}
g_{0}^{L}(x)= & \sum_{j=1, j \neq p}^{n} c_{0 j} x_{j}+c_{0 p} x_{p}+\delta_{0} \\
\geq & \sum_{j=1, j \neq p, c_{0 j}>0}^{n} c_{0 j} l_{j}+\sum_{j=1, j \neq p, c_{0 j}<0}^{n} c_{i j} u_{j} \\
& +\delta_{0}+c_{0 p} x_{p} \\
= & R g_{0 p}^{L}+c_{0 p} x_{p} \\
> & R g_{0 p}^{L}+\bar{g}-R g_{0 p}^{L} \\
= & \bar{g} .
\end{aligned}
$$

Thus, we have

$$
g_{0}(x) \geq g_{0}^{L}(x)>\bar{g} .
$$

Therefore, there does not exist global optimal solution of the $\mathrm{GQP}\left(X^{0}\right)$ over $\overline{\bar{X}}$.

Similarly, if $c_{0 p}<0$, there is no global optimal solution of problem GQP $\left(X^{0}\right)$ over $\underline{X}$.

Theorem 3. For any subrectangle $X \subseteq X^{0}$, for all $i=1, \ldots, m$, the following conclusions hold.

(i) If $R L B_{i}>b_{i}$ for some $i \in\{1, \ldots, m\}$, then there exists no global optimal solution of the $G Q P\left(X^{0}\right)$ over $X$.

(ii) If $R L B_{i} \leq b_{i}$ for each $i \in\{1, \ldots, m\}$, then, for each $p \in\{1,2, \ldots, n\}$, if $c_{i p}>0$, then there is no global optimal solution of the $G Q P\left(X^{0}\right)$ over $\widetilde{X}=\left(\widetilde{X}_{j}\right)_{n \times 1}$; if $c_{i p}<0$, there does not exist global optimal solution of the $G Q P\left(X^{0}\right)$ over $\widehat{X}=\left(\widehat{X}_{j}\right)_{n \times 1}$, where

$$
\begin{aligned}
& \widetilde{X}_{j}= \begin{cases}X_{j}, & j \neq p, j=1, \ldots, n, \\
\left(\mu_{i p}, u_{p}\right] \bigcap X_{p}, & j=p ;\end{cases} \\
& \widehat{X}_{j}= \begin{cases}X_{j}, & j \neq p, j=1, \ldots, n, \\
{\left[l_{p}, \mu_{i p}\right) \bigcap X_{p},} & j=p .\end{cases}
\end{aligned}
$$

Proof. (i) If $\mathrm{RLB}_{i}>b_{i}$ for some $i \in\{1, \ldots, m\}$, then for all $x \in X$, we have

$$
g_{i}(x) \geq g_{i}^{L}(x) \geq \mathrm{RLB}_{i}>b_{i} .
$$

Therefore, there exists no global optimal solution of the $\mathrm{GQP}\left(X^{0}\right)$ over $X$.

(ii) If $\mathrm{RLB}_{i} \leq b_{i}$ for each $i \in\{1, \ldots, m\}$, then, for each $p \in\{1,2, \ldots, n\}$, if $c_{i p}>0$, for all $x \in \widetilde{X}$, we have $x_{p}>\mu_{i p}$; that is,

$$
x_{p}>\frac{b_{i}-R g_{i p}^{L}}{c_{i p}} \text { or } \quad c_{i p} x_{p}>b_{i}-R g_{i p}^{L} .
$$

Therefore, we get that

$$
\begin{aligned}
g_{i}^{L}(x)= & \sum_{j=1, j \neq p}^{n} c_{i j} x_{j}+c_{i p} x_{p}+\delta_{i} \\
\geq & \sum_{j=1, j \neq p, c_{i j}>0}^{n} c_{i j} l_{j} \\
& +\sum_{j=1, j \neq p, c_{i j}<0}^{n} c_{i j} u_{j}+\delta_{i}+c_{i p} x_{p} \\
= & R g_{i p}^{L}+c_{i p} x_{p} \\
> & R g_{i p}^{L}+b_{i}-R g_{i p}^{L} \\
= & b_{i} .
\end{aligned}
$$

Thus, we have

$$
g_{i}(x) \geq g_{i}^{L}(x)>b_{i} .
$$

Hence, there does not exist global optimal solution of the $\operatorname{GQP}\left(X^{0}\right)$ over $\widetilde{X}$.

Similarly, if $c_{i p}<0$, there is no global optimal solution of the $\operatorname{GQP}\left(X^{0}\right)$ over $\widehat{X}$.

By Theorems 2 and 3, we can give a range reduction technique to reject some regions where there does not exist the global optimal solution of the $\operatorname{GQP}\left(X^{0}\right)$. Let $X=\left(X_{j}\right)_{n \times 1}$ with $X_{j}=\left[l_{j}, u_{j}\right](j=1, \ldots, n)$ be any subrectangle of $X^{0}$.

\section{Range Reduction Technique}

Reduction Rule (i). Calculate $\mathrm{RLB}_{0}$.

If $\mathrm{RLB}_{0}>\bar{g}$, then let $X=\emptyset$; otherwise, calculate $R g_{0 p}^{L}$ and $\gamma_{p}(p=1, \ldots, n)$.

If $c_{0 p}>0$ and $\gamma_{p}<u_{p}$ for some $p \in\{1, \ldots, n\}$, then let $u_{p}=\gamma_{p}$ and $X=\left(X_{j}\right)_{n \times 1}$ with $X_{j}=\left[l_{j}, u_{j}\right](j=1, \ldots, n)$.

If $c_{0 p}<0$ and $\gamma_{p}>l_{p}$ for some $p \in\{1, \ldots, n\}$, then let $l_{p}=\gamma_{p}$ and $X=\left(X_{j}\right)_{n \times 1}$ with $X_{j}=\left[l_{j}, u_{j}\right](j=1, \ldots, n)$.

Reduction Rule (ii). For each $i=1, \ldots, M$, calculate $\mathrm{RLB}_{i}$.

If $\mathrm{RLB}_{i}>b_{i}$ for some $i \in\{1, \ldots, M\}$, let $X=\emptyset$; otherwise, calculate $R g_{i p}^{L}$ and $\mu_{i p}(i=1, \ldots, M, p=1, \ldots, n)$.

If $c_{i p}>0$ and $\mu_{i p}<u_{p}$ for some $i \in\{1, \ldots, M\}$ and $p \in$ $\{1, \ldots, n\}$, then let $u_{p}=\mu_{i p}$ and $X=\left(X_{j}\right)_{n \times 1}$ with $X_{j}=\left[l_{j}, u_{j}\right]$ $(j=1, \ldots, n)$.

If $c_{0 p}<0$ and $\mu_{i p}>l_{p}$ for some $i \in\{1, \ldots, M\}$ and $p \in$ $\{1, \ldots, n\}$, then let $l_{p}=\mu_{i p}$ and $X=\left(X_{j}\right)_{n \times 1}$ with $X_{j}=\left[l_{j}, u_{j}\right]$ $(j=1, \ldots, n)$.

3.2. Branch-Reduction-Bound Algorithm. Let $\mathrm{LB}\left(X^{k}\right)$ refer to the optimal value of the (RLP) over the subhyperrectangles $X^{k}$ and let $x^{k}=x\left(X^{k}\right)$ refer to an element of corresponding argmin. Combining the former relaxation linear programming problem, branching operation, and range reduction technique together, the basic steps of the proposed algorithm for globally solving the (GQP) may be stated as follows. 


\section{Algorithm 4.}

Step 0 (initialization). Let the iteration counter $k=0$, the set of all active nodes $\Delta_{0}=\left\{X^{0}\right\}$, and the convergence tolerance $\epsilon>0$. Let the initial upper bound $\bar{g}=+\infty$, and the set of feasible points $F=\emptyset$.

Solve the $\operatorname{RLP}\left(X^{0}\right)$, obtain optimal solution $x^{0}=x\left(X^{0}\right)$ and optimal value $\operatorname{LB}\left(X^{0}\right)$ for the $\operatorname{RLP}\left(X^{0}\right)$, and let $\operatorname{LB}_{0}=$ $\mathrm{LB}\left(X^{0}\right)$. If $x^{0}$ is feasible to the $\operatorname{GQP}\left(X^{0}\right)$, let $\bar{g}=g_{0}\left(x^{0}\right)$ and $F=F \cup\left\{x^{0}\right\}$. If $\bar{g}-\mathrm{LB}_{0} \leq \epsilon$, then stop with $x^{0}$ as the global $\epsilon$-optimal solution for the $\mathrm{GQP}\left(X^{0}\right)$. Otherwise, proceed to Step 1.

Step 1 (branching). Using the proposed branching rule to partition $X^{k}$ into two new subhyperrectangles, and denote the set of new partition rectangles by $\bar{X}^{k}$.

Step 2 (reduction). For each subhyperrectangle $X \in \bar{X}^{k}$, utilize the proposed range reduction technique to reject a part of the region or the whole region $X$, and still denote the remaining region by $X$, and still denote the remaining partition set by $\bar{X}^{k}$.

Step 3 (updating the upper bound). If $\bar{X}^{k} \neq \emptyset$, solve the $\operatorname{RLP}(X)$ to obtain $\operatorname{LB}(X)$ and $x(X)$ for each $X \in \bar{X}^{k}$. For each $X \in \bar{X}^{k}$, if $\operatorname{LB}(X)>\bar{g}$, set $\bar{X}^{k}:=\bar{X}^{k} \backslash X$; otherwise, select the midpoint $x^{\text {mid }}$ of $X$; if $x^{\text {mid }}$ is feasible to the $\operatorname{GQP}\left(X^{0}\right)$, then let $F:=F \cup\left\{x^{\mathrm{mid}}\right\}$, and if $x(X)$ is feasible to the $\operatorname{GQP}\left(X^{0}\right)$, then let $F:=F \cup\{x(X)\}$.

If $F \neq \emptyset$, update the upper bound $\bar{g}:=\min _{x \in F} g_{0}(x)$, and denote the best known feasible point by $x^{*}:=$ $\operatorname{argmin}_{x \in F} g_{0}(x)$.

Step 4 (updating the lower bound). The partition set remaining is now $\Delta_{k}:=\left(\Delta_{k} \backslash X^{k}\right) \cup \bar{X}^{k}$, and update the lower bound $\mathrm{LB}_{k}:=\inf _{X \in \Delta_{k}} \mathrm{LB}(X)$.

Step 5 (convergence checking). Set $\Delta_{k+1}=\Delta_{k} \backslash\{X: \bar{g}-$ $\left.\operatorname{LB}(X) \leq \epsilon, X \in \Delta_{k}\right\}$. If $\Delta_{k+1}=\emptyset$, then stop with $\bar{g}$ as the global optimal value of the (GQP) and $x^{*}$ as the global optimal solution. Otherwise, select an active node $X^{k+1}$ such that $X^{k+1}=\operatorname{argmin}_{X \in \Delta_{k+1}} \operatorname{LB}(X), x^{k+1}:=x\left(X^{k+1}\right)$. Set $k:=$ $k+1$, and return to Step 1 .

3.3. Convergence Analysis. In this subsection, the global convergence properties of the proposed algorithm are presented. If the algorithm does not terminate finitely, then the branching rule guarantees all the intervals shrinking to a singleton for all the variables. On the other hand, Theorem 1 guarantees that as $\left\|u^{k}-l^{k}\right\| \rightarrow 0$ the relaxation linear programming problem $\operatorname{RLP}\left(X^{k}\right)$ approaches $\operatorname{GQP}\left(X^{k}\right)$. So it is not surprising that the algorithm is shown to be the convergent to the global optimal solution.

Theorem 5. The proposed algorithm either terminates finitely with the global $\epsilon$-optimal solution $x^{*}$ of problem $G Q P\left(X^{0}\right)$ or generates an infinite sequence of iteration such that along any infinite branch of the branch and bound tree, any accumulation point of the sequence $\left\{x^{k}\right\}$ will be the global solution of problem $G Q P\left(X^{0}\right)$.

Proof. If the algorithm is finite, suppose that it terminates in iteration $k, k \geq 0$. Upon termination, we have

$$
\bar{g}-\mathrm{LB}_{k} \leq \epsilon
$$

By Steps 0 and 3 in Algorithm 4, we can find a feasible solution $x^{*}$ for the problem $\operatorname{GQP}\left(X^{0}\right)$ such that $g_{0}\left(x^{*}\right)=\bar{g}$, which implies

$$
g_{0}\left(x^{*}\right)-\mathrm{LB}_{k} \leq \epsilon
$$

Set $v$ denotes the optimal value of problem $\mathrm{GQP}\left(X^{0}\right)$; then, by computational method of lower bound, we have

$$
\mathrm{LB}_{k} \leq v
$$

Since $x^{*}$ is a feasible solution of problem $\operatorname{GQP}\left(X^{0}\right)$, we have

$$
v \leq g_{0}\left(x^{*}\right)
$$

By inequalities (26)-(28), we can get that

$$
v \leq g_{0}\left(x^{*}\right) \leq \mathrm{LB}_{k}+\epsilon \leq v+\epsilon .
$$

That is,

$$
v \leq g_{0}\left(x^{*}\right) \leq v+\epsilon .
$$

Therefore, $x^{*}$ is a global $\epsilon$-optimal solution of the problem $\operatorname{GQP}\left(X^{0}\right)$.

If the algorithm is infinite, by the proposed algorithm, we know that the lower bound sequence $\left\{\mathrm{LB}_{k}\right\}$ is nondecreasing and bounded by the upper bound $\min _{x \in F} g_{0}(x)$; therefore, there exists limitation such that

$$
\mathrm{LB}=\lim _{k \rightarrow \infty} \mathrm{LB}_{k} \leq \min _{x \in F} g_{0}(x)
$$

Since $\left\{x^{k}\right\} \in X^{0}$, where $X^{0}$ is a bounded close set, there exists a convergent subsequence $\left\{x^{\beta}\right\} \subseteq\left\{x^{k}\right\}$ and assume $\lim _{\beta \rightarrow \infty} x^{\beta}=x^{*}$. Then by the proposed algorithm, there exists a decreasing subsequence $\left\{X^{\sigma}\right\} \subseteq X^{\beta}$ where $X^{\beta} \in \Delta_{\beta}$ with $x^{\sigma} \in X^{\sigma}, \mathrm{LB}_{\sigma}=\mathrm{LB}\left(X^{\sigma}\right)=g_{0}^{L}\left(x^{\sigma}\right)$ and $\lim _{\sigma \rightarrow \infty} X^{\sigma}=$ $\left\{x^{*}\right\}$. By Theorem 1 and the continuity of the function $g_{0}(x)$, we can follow that

$$
\lim _{\sigma \rightarrow \infty} \mathrm{LB}_{\sigma}=\lim _{\sigma \rightarrow \infty} g_{0}^{L}\left(x^{\sigma}\right)=\lim _{\sigma \rightarrow \infty} g_{0}\left(x^{\sigma}\right)=g_{0}\left(x^{*}\right)
$$


TABLE 1: Numerical results for the test Examples 6-13.

\begin{tabular}{|c|c|c|c|}
\hline Example & References & Optimal value & Optimal solution \\
\hline \multirow{2}{*}{6} & [ours] & -16.000000000 & $(5.000000000,1.000000000)$ \\
\hline & {$[15]$} & -16.0 & $(5.0,1.0)$ \\
\hline \multirow{4}{*}{7} & [ours] & 6.777777779 & $(2.000000000,1.666666667)$ \\
\hline & {$[16]$} & 6.7780 & $(2.00003,1.66665)$ \\
\hline & {$[13]$} & 6.777782016 & $(2.000000000,1.666666667)$ \\
\hline & {$[13]$} & 6.777781963 & $(2.000000000,1.666666667)$ \\
\hline \multirow{5}{*}{8} & [ours] & 0.500000442 & $(0.500000000,0.500000000)$ \\
\hline & {$[16]$} & 0.5 & $(0.5,0.5)$ \\
\hline & [17] & 0.5 & $(0.5,0.5)$ \\
\hline & {$[13]$} & 0.500004627 & $\left(\begin{array}{lll}0.5 & 0.5)\end{array}\right.$ \\
\hline & {$[13]$} & 0.5 & $(0.5,0.5)$ \\
\hline \multirow{2}{*}{9} & [ours] & 118.383671904 & $(2.555745855,3.130201688)$ \\
\hline & {$[18]$} & 118.383756475281 & $(2.5557793695324,3.13016463929768)$ \\
\hline \multirow{2}{*}{10} & [ours] & -1.162882693 & $(1.500000000,1.500000000)$ \\
\hline & {$[19]$} & -1.16288 & $(1.5,1.5)$ \\
\hline \multirow{2}{*}{11} & [ours] & 1.177125051 & $(1.177124344,2.177124344)$ \\
\hline & {$[14]$} & 1.177124327 & $(1.177124327,2.177124353)$ \\
\hline \multirow{2}{*}{12} & [ours] & -0.999999410 & $(2.000000000,1.000000000)$ \\
\hline & {$[14]$} & -1.0 & $(2.000000,1.000000)$ \\
\hline \multirow{2}{*}{13} & [ours] & -11.363636364 & $(1.000000000,0.181818470,0.983332113)$ \\
\hline & {$[20]$} & -10.35 & $(0.998712,0.196213,0.979216)$ \\
\hline
\end{tabular}

Then all remaining is to demonstrate that $x^{*}$ is feasible to the $\operatorname{GQP}\left(X^{0}\right)$.

Firstly, since $X^{0}$ is bounded and closed set, obviously we have $x^{*} \in X^{0}$.

Secondly, we will prove that $g_{i}\left(x^{*}\right) \leq b_{i}, i=1, \ldots, m$.

By contradiction, assume $g_{\tau}\left(x^{*}\right)>b_{\tau}$ for some $\tau \in$ $\{1, \ldots, m\}$. Since $g_{\tau}^{L}(x)$ is a continuous function and by Theorem 1 , the sequence $\left\{g_{\tau}^{L}\left(x^{\sigma}\right)\right\}$ is convergent to $g_{\tau}\left(x^{*}\right)$; that is, there exists a $\bar{\sigma}$ such that

$$
\left|g_{\tau}^{L}\left(x^{\sigma}\right)-g_{\tau}\left(x^{*}\right)\right|<g_{\tau}\left(x^{*}\right)-b_{\tau} \quad \text { for any } \sigma>\bar{\sigma} .
$$

Hence, for any $\sigma>\bar{\sigma}$, we have $g_{\tau}^{L}\left(x^{\sigma}\right)>b_{\tau}$, which shows that the $\operatorname{LRP}\left(X^{\sigma}\right)$ is infeasible and violates the assumption that $x^{\sigma}=x\left(X^{\sigma}\right)$. This will bring about a contradiction; thus, the proof is competed.

\section{Numerical Experiments}

To compare the proposed algorithm with the known methods in computational efficiency and solution quality, some test examples in recent literature are implemented on microcomputer, the algorithm procedure is coded in $\mathrm{C}++$, and each relaxation linear programming is solved by using simplex method, and the convergence tolerance $\epsilon$ is set to $10^{-6}$ in Examples 6-13 and $10^{-5}$ in Example 14, respectively. These test examples and numerical results compared with the known algorithms are given in Tables 1 and 2 .
TABLE 2: Numerical results for the test Examples 6-13.

\begin{tabular}{|c|c|c|c|}
\hline Example & References & $\begin{array}{c}\text { Number of } \\
\text { algorithm iteration }\end{array}$ & $\begin{array}{c}\text { Computational } \\
\text { times }\end{array}$ \\
\hline \multirow{2}{*}{6} & [ours] & 5 & \multirow{2}{*}{0.00184588} \\
\hline & {$[15]$} & 10 & \\
\hline \multirow{4}{*}{7} & [ours] & 10 & 0.00266479 \\
\hline & {$[16]$} & 44 & 0.18 \\
\hline & {$[13]$} & 40 & 0.032 \\
\hline & [13] & 32 & 0.015 \\
\hline \multirow{5}{*}{8} & [ours] & 37 & 0.0192625 \\
\hline & {$[16]$} & 91 & 0.85 \\
\hline & [17] & 96 & 1 \\
\hline & [13] & 34 & 0.056 \\
\hline & [13] & 29 & 0.041 \\
\hline \multirow{2}{*}{9} & [ours] & 59 & 0.0385038 \\
\hline & {$[18]$} & 210 & 0.78 \\
\hline \multirow{2}{*}{10} & [ours] & 24 & \multirow{2}{*}{0.0105913} \\
\hline & [19] & 84 & \\
\hline \multirow{2}{*}{11} & [ours] & 22 & 0.00919099 \\
\hline & {$[14]$} & 434 & 1 \\
\hline \multirow{2}{*}{12} & [ours] & 21 & 0.00849446 \\
\hline & {$[14]$} & 24 & $<1$ \\
\hline \multirow{2}{*}{13} & [ours] & 420 & 0.284541 \\
\hline & {$[20]$} & 1648 & 0.3438 \\
\hline
\end{tabular}


TABle 3: Numerical results for Example 14.

\begin{tabular}{|c|c|c|c|c|c|}
\hline References & Dimension of variable & $n=5$ & $n=10$ & $n=20$ & $n=30$ \\
\hline \multirow{2}{*}{ This paper } & CPU time(s) & 0.0181791 & 0.302157 & 6.01095 & 44.4965 \\
\hline & Number of iteration & 12 & 32 & 88 & 206 \\
\hline \multirow[t]{2}{*}{ Ref. [21] } & CPU time(s) & 10.11 & 21.86 & 47.00 & 106.33 \\
\hline & Number of iteration & 141 & 283 & 651 & 965 \\
\hline
\end{tabular}

Example 6 (see [15]). Consider

$$
\begin{array}{ll}
\min & -x_{1}^{2}+x_{1} x_{2}+x_{2}^{2}+x_{1}-2 x_{2} \\
\text { s.t. } & x_{1}+x_{2} \leq 6 \\
& -2 x_{1}^{2}+x_{2}^{2}+2 x_{1}+x_{2} \leq-4, \\
& 1 \leq x_{1} \leq 6, \quad 1 \leq x_{2} \leq 6
\end{array}
$$

Example 7 (see $[13,16])$. Consider

$$
\begin{array}{ll}
\min & x_{1}^{2}+x_{2}^{2} \\
\text { s.t. } & 0.3 x_{1} x_{2} \geq 1, \quad 2 \leq x_{1} \leq 5,1 \leq x_{2} \leq 3
\end{array}
$$

Example 8 (see $[13,16,17])$. Consider

$$
\begin{array}{ll}
\min & x_{1} \\
\text { s.t. } & 4 x_{2}-4 x_{1}^{2} \leq 1 \\
& -x_{1}-x_{2} \leq-1, \\
& 0.01 \leq x_{1} \leq 15, \\
& 0.01 \leq x_{2} \leq 15 .
\end{array}
$$

Example 9 (see [18]). Consider

$$
\begin{array}{ll}
\min & 6 x_{1}^{2}+4 x_{2}^{2}+5 x_{1} x_{2} \\
\text { s.t. } & -6 x_{1} x_{2} \leq-48 \\
& 0 \leq x_{1} \leq 10 \\
& 0 \leq x_{2} \leq 10
\end{array}
$$

Example 10 (see [19]). Consider

$$
\begin{array}{ll}
\min & -x_{1}+x_{1} x_{2}^{0.5}-x_{2} \\
\text { s.t. } & -6 x_{1}+8 x_{2} \leq 3, \\
& 3 x_{1}-x_{2} \leq 3, \\
& 1 \leq x_{1} \leq 1.5 \\
& 1 \leq x_{2} \leq 1.5
\end{array}
$$

Example 11 (see [14]). Consider

$$
\begin{array}{ll}
\min & x_{1} \\
\text { s.t. } & \frac{1}{4} x_{1}+\frac{1}{2} x_{2}-\frac{1}{16} x_{1}^{2}-\frac{1}{16} x_{2}^{2} \leq 1, \\
& \frac{1}{14} x_{1}^{2}+\frac{1}{14} x_{2}^{2}-\frac{3}{7} x_{1}-\frac{3}{7} x_{2} \leq-1, \\
& 1 \leq x_{1} \leq 5.5, \quad 1 \leq x_{2} \leq 5.5 .
\end{array}
$$

Example 12 (see [14]). Consider

$$
\begin{array}{ll}
\min & x_{1} x_{2}-2 x_{1}+x_{2}+1 \\
\text { s.t. } & 8 x_{2}^{2}-6 x_{1}-16 x_{2} \leq-11, \\
& -x_{2}^{2}+3 x_{1}+2 x_{2} \leq 7 \\
& 1 \leq x_{1} \leq 2.5 \\
& 1 \leq x_{2} \leq 2.225
\end{array}
$$

Example 13 (see [20]). Consider

$$
\begin{array}{ll}
\min & -4 x_{2}+\left(x_{1}-1\right)^{2}+x_{2}^{2}-10 x_{3}^{2} \\
\text { s.t. } & x_{1}^{2}+x_{2}^{2}+x_{3}^{2} \leq 2, \\
& \left(x_{1}-2\right)^{2}+x_{2}^{2}+x_{3}^{2} \leq 2, \\
& 2-\sqrt{2} \leq x_{1} \leq \sqrt{2}, \\
& 0 \leq x_{2}, \quad x_{3} \leq \sqrt{2} .
\end{array}
$$

Example 14 (see [21]). Consider

$$
\begin{array}{ll}
\min & -\sum_{i=1}^{n} x_{i}^{2} \\
\text { s.t. } & \sum_{i=1}^{j} x_{i} \leq j, \quad j \in\{1,2, \ldots, n\}, \\
& x_{i} \geq 0, \quad i \in\{1,2, \ldots, n\} .
\end{array}
$$

The numerical results for Example 14 compared with [21] are given in Table 3 .

The numerical results for test Examples 6-14 show that the proposed algorithm is robust and can be used to globally solve generalized quadratic programming (GQP). 


\section{Concluding Remarks}

In this paper, a global optimization algorithm based on a new linearizing method and a range reduction technique is presented for solving the (GQP). The relaxation linear programming of the original problem (GQP) is established by underestimating the objective and constraint functions with linear functions. By making full use of the currently known upper bound and relaxation linear programming of the (GQP), a range reduction technique is presented. The algorithm is convergent to the global minimum through the successive refinement of a linear relaxation of the feasible region and/or of the objective function and the subsequent solution of a series of relaxation linear programming problems. Numerical results show effectiveness and robustness of the proposed algorithm.

\section{Acknowledgments}

This paper is supported by the National Natural Science Foundation of China (11171094), the Foundation for University Key Teacher by the Ministry of Education of Henan Province (2010GGJS-140), and the Natural Science Foundation of Henan Educational Committee (2010B110010). The authors acknowledged the help of Yingfeng Zhao while preparing the paper.

\section{References}

[1] M. H. Khammash, "Synthesis of globally optimal controllers for robust performance to unstructured uncertainty," IEEE Transactions on Automatic Control, vol. 41, no. 2, pp. 189-198, 1996.

[2] M. H. Khammash, M. V. Salapaka, and T. Vanvoorhis, "Synthesis of globally optimal controllers in $l_{1}$ using linear relaxation," in Proceedings of the 37th IEEE Conference on Decision and Control (CDC '98), pp. 3315-3320, December 1998.

[3] W. A. Lodwick, "Preprocessing nonlinear functional constraints with applications to the pooling problem," ORSA Journal on Computing, vol. 4, no. 2, pp. 119-131, 1992.

[4] C. A. Floudas and V. Visweswaran, "Primal-relaxed dual global optimization approach," Journal of Optimization Theory and Applications, vol. 78, no. 2, pp. 187-225, 1993.

[5] D. Vandenbussche and G. L. Nemhauser, "A branch-andcut algorithm for nonconvex quadratic programs with box constraints," Mathematical Programming A, vol. 102, no. 3, pp. 559-575, 2005.

[6] R. Cambini and C. Sodini, "Decomposition methods for solving nonconvex quadratic programs via branch and bound," Journal of Global Optimization, vol. 33, no. 3, pp. 313-336, 2005.

[7] H.-M. Li and K.-C. Zhang, "A decomposition algorithm for solving large-scale quadratic programming problems," Applied Mathematics and Computation, vol. 173, no. 1, pp. 394-403, 2006.

[8] L. T. H. An, "An efficient algorithm for globally minimizing a quadratic function under convex quadratic constraints," Mathematical Programming A, vol. 87, no. 3, pp. 401-426, 2000.

[9] Y. Ye, "Approximating global quadratic optimization with convex quadratic constraints," Journal of Global Optimization, vol. 15, no. 1, pp. 1-17, 1999.
[10] T. V. Voorhis, "A global optimization algorithm using Lagrangian underestimates and the interval Newton method," Journal of Global Optimization, vol. 24, no. 3, pp. 349-370, 2002.

[11] H. D. Sherali and C. H. Tuncbilek, "A global optimization algorithm for polynomial programming problems using a reformulation-linearization technique," Journal of Global Optimization, vol. 2, no. 1, pp. 101-112, 1992.

[12] S.-J. Qu, K.-C. Zhang, and Y. Ji, "A global optimization algorithm using parametric linearization relaxation," Applied Mathematics and Computation, vol. 186, no. 1, pp. 763-771, 2007.

[13] P. P. Shen and L. M. Liu, "A global optimization approach to quadratic programming problems with nonconvex quadratic constraints," Chinese Journal of Engineering Mathematics, vol. 25, no. 5, pp. 923-926, 2008.

[14] P. Shen and H. Jiao, "A new rectangle branch-and-pruning approach for generalized geometric programming," Applied Mathematics and Computation, vol. 183, no. 2, pp. 1027-1038, 2006.

[15] N. V. Thoai, "Duality bound method for the general quadratic programming problem with quadratic constraints," Journal of Optimization Theory and Applications, vol. 107, no. 2, pp. 331354, 2000.

[16] Y. Wang and Z. Liang, "A deterministic global optimization algorithm for generalized geometric programming," Applied Mathematics and Computation, vol. 168, no. 1, pp. 722-737, 2005.

[17] Y. Wang, K. Zhang, and Y. Gao, "Global optimization of generalized geometric programming," Computers \& Mathematics with Applications, vol. 48, no. 10-11, pp. 1505-1516, 2004.

[18] Y. Gao, Y. Shang, and L. Zhang, "A branch and reduce approach for solving nonconvex quadratic programming problems with quadratic constraints," OR Transactions, vol. 9, no. 2, pp. 9-20, 2005.

[19] P. Shen, "Linearization method of global optimization for generalized geometric programming," Applied Mathematics and Computation, vol. 162, no. 1, pp. 353-370, 2005.

[20] P. Shen and X. Li, "Branch-reduction-bound algorithm for generalized geometric programming," Journal of Global Optimization, vol. 56, no. 3, pp. 1123-1142, 2013.

[21] Y. Gao, H. Xue, and P. Shen, "A new rectangle branch-andreduce approach for solving nonconvex quadratic programming problems," Applied Mathematics and Computation, vol. 168, no. 2, pp. 1409-1418, 2005. 


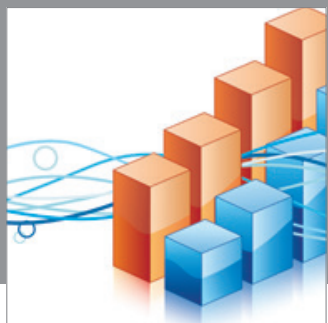

Advances in

Operations Research

mansans

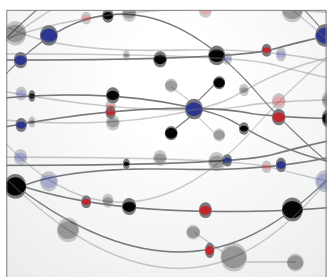

The Scientific World Journal
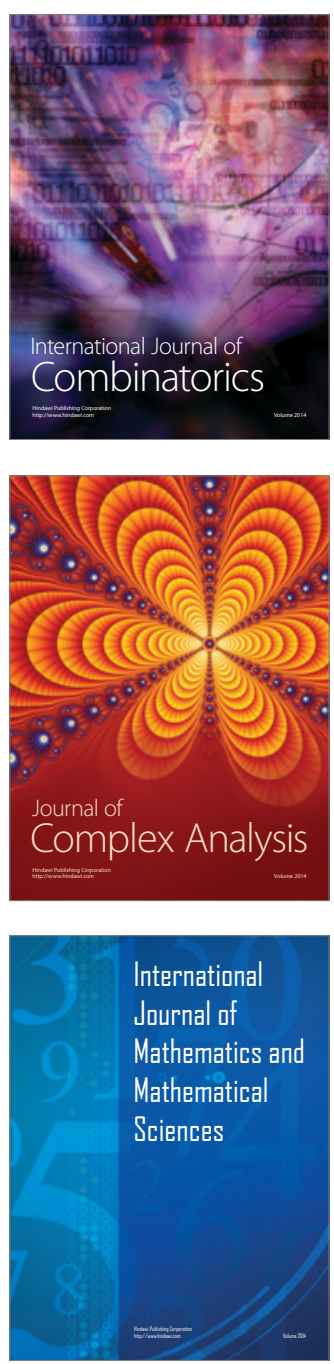
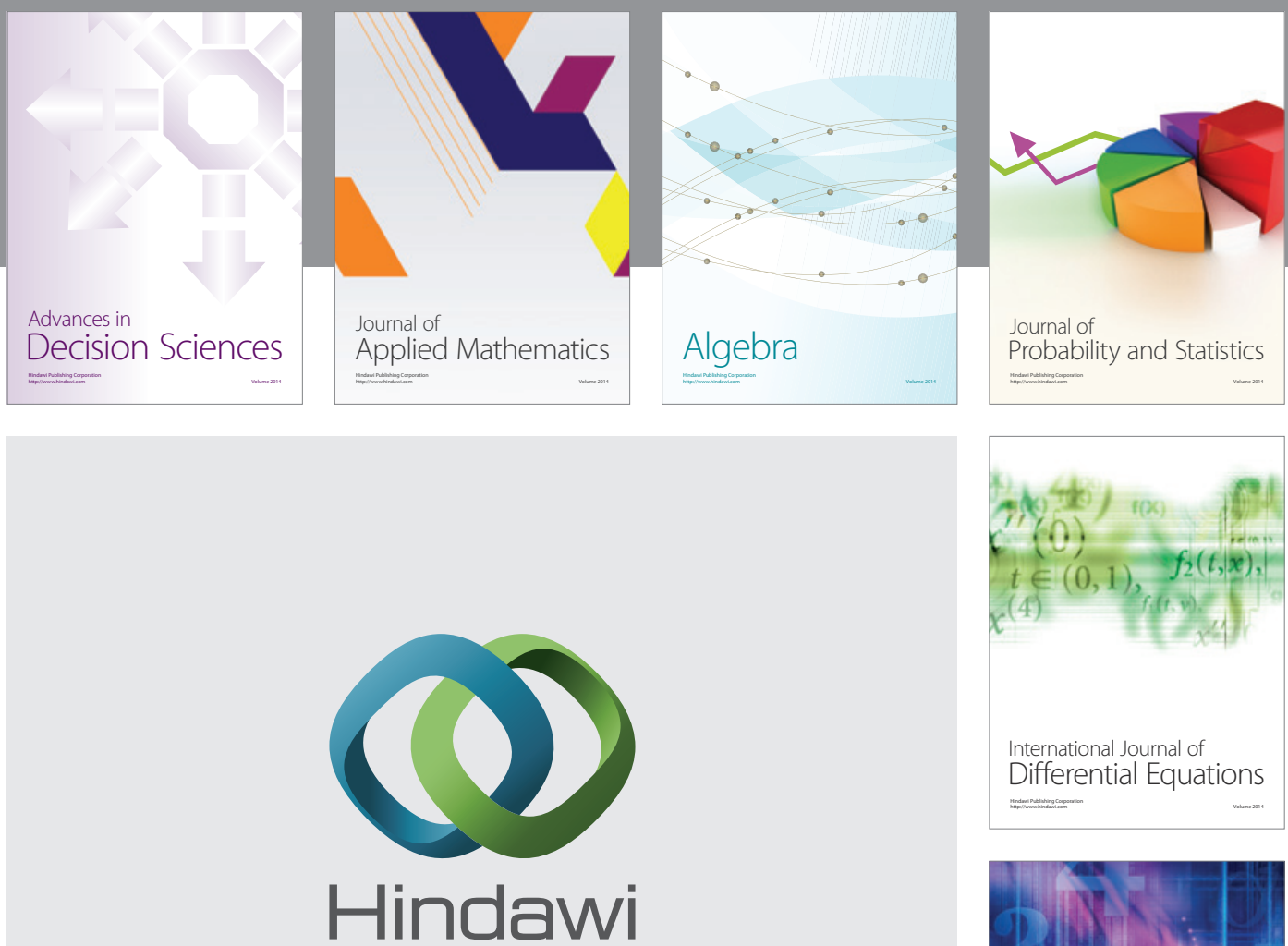

Submit your manuscripts at http://www.hindawi.com
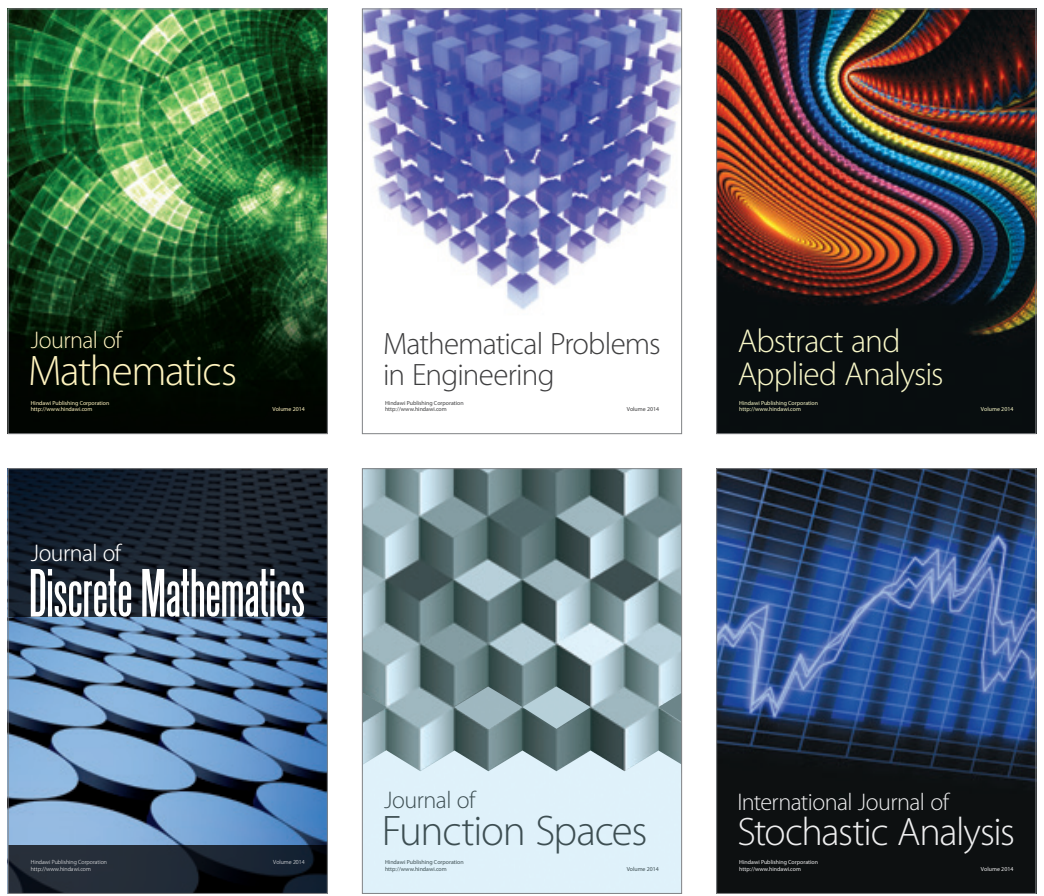

Journal of

Function Spaces

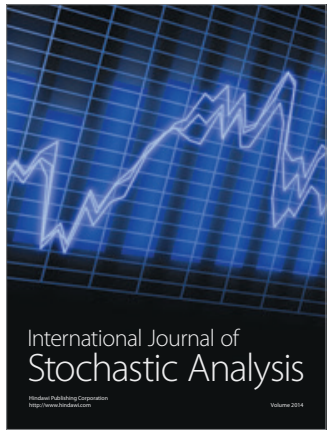

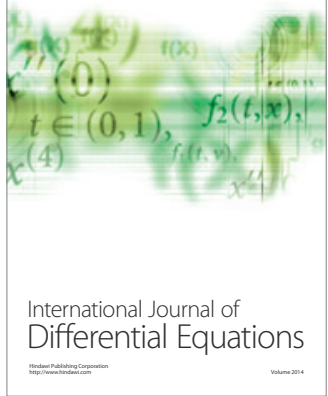
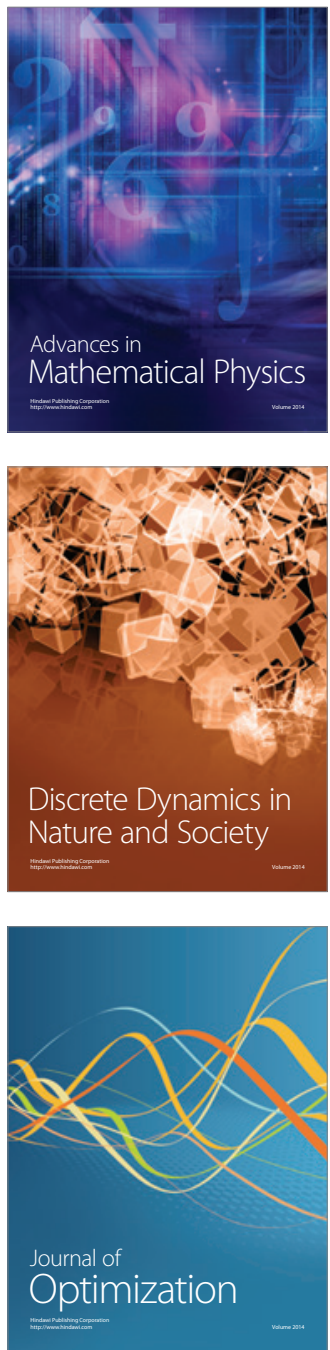